Document downloaded from:

http://hdl.handle.net/10251/82086

This paper must be cited as:

Rosso, P.; Bosco, C.; Damiano, R.; Patti, V.; Cambria, E. (2016). Emotion and Sentiment in Social and Expressive Media: Introduction to the special issue. Information Processing and Management. 52(1):1-4. doi:10.1016/j.ipm.2015.11.002.

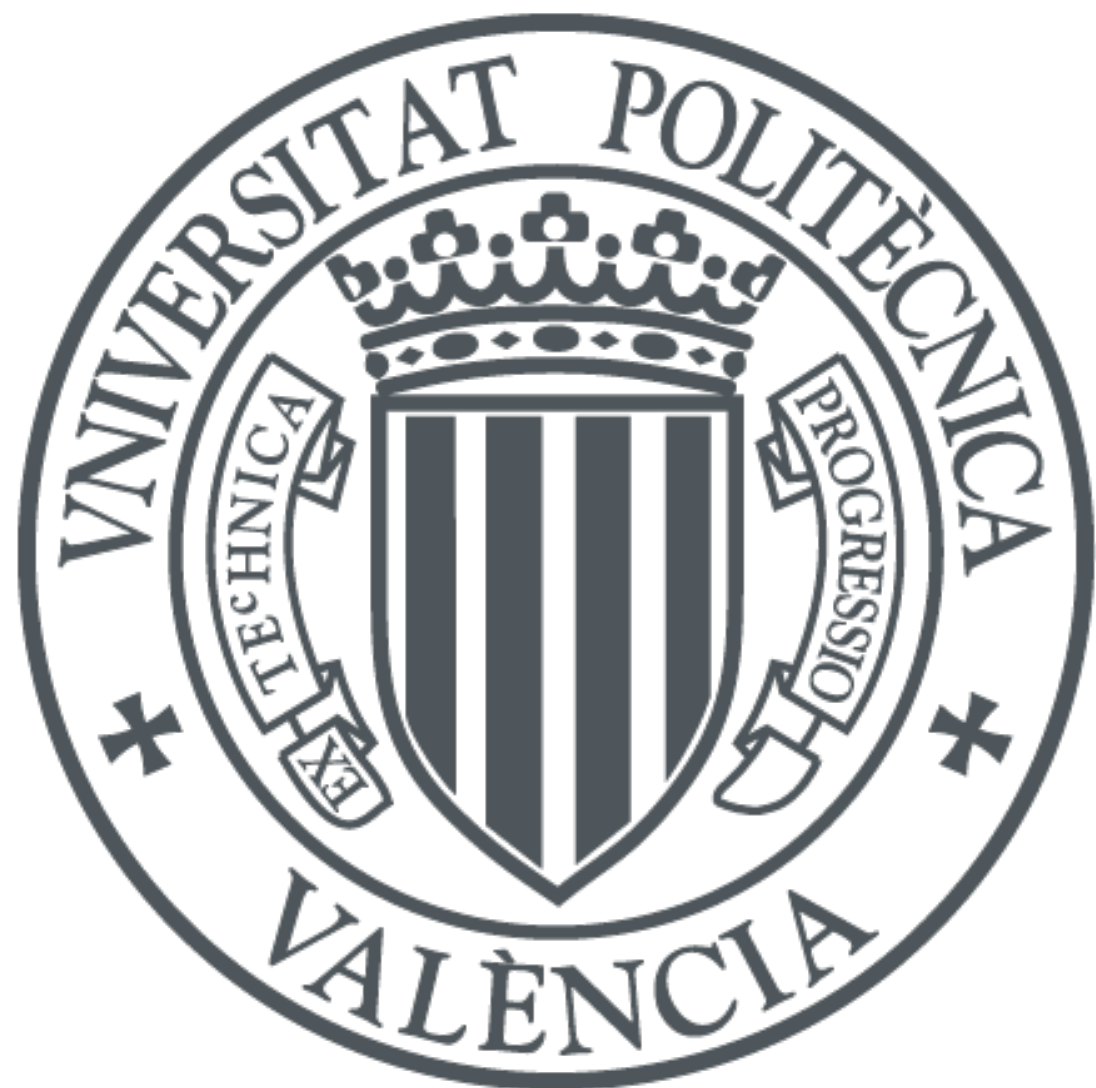

The final publication is available at

http://dx.doi.org/10.1016/j.ipm.2015.11.002

Copyright Elsevier

Additional Information

This is the author's version of a work that was accepted for publication in Information Processing and Management. Changes resulting from the publishing process, such as peer review, editing, corrections, structural formatting, and other quality control mechanisms may not be reflected in this document. Changes may have been made to this work since it was submitted for publication. A definitive version was subsequently published in Information Processing and Management 52 (2016) 1-4. DOI 10.1016/j.ipm.2015.11.002 


\title{
Emotion and sentiment in social and expressive media: introduction to the special issue
}

\author{
Paolo Rosso $^{\mathrm{a}}$, Cristina Bosco ${ }^{\mathrm{b}}$, Erik Cambria ${ }^{\mathrm{c}}$, Rossana Damiano ${ }^{\mathrm{b}}$, Viviana \\ Patti $^{\mathrm{b}}$ \\ ${ }^{a}$ NLE Lab, PRHLT Research Center \\ Universitat Politècnica de València, Spain \\ ${ }^{b}$ Dipartimento di Informatica, University of Turin, Italy \\ ${ }^{c}$ Nanyang Technological University, Singapore
}

Keywords: sentiment analysis, affective processing, social media, expressive media

\section{Introduction}

Social and expressive media represent a challenge and a push forward for research on emotion and sentiment analysis. The advent of social media has brought about new paradigms of interactions that foster first-person engagement and crowdsourced contents: the subjective dimension moves to the foreground, opening the way to the emergence of an affective component within a dynamic corpus of digitized contents created and enriched by the users. Expressive media, which play a key role in fields related to creativity, such as figurative arts, music or drama, gather multimedia contents into online social environments, by joining the social dimension with the aims of artistic creation and self-expression. Artistic creation and performance seem to be a very interesting testbed for cross-validating and possibly integrating approaches, models and tools for automatically analyzing emotion and sentiment. In fact, in such contexts the social and affective dimensions (emotions and feelings) naturally emerges (Silvia, 2005), think for instance of the visitors' feedback to a real or virtual art exhibition, or of the audience-performance interaction.

Social and expressive media generate - in a continuous loop of interaction between online environments and the community of users - new digitized contents, including spontaneous, multi-faceted and unstructured user responses. This advocates new techniques for automatic processing, indexing and retrieval of the affective and subjective information conveyed. The automatic analysis of the opinions and of the affective component expressed in such data, in fact,

Email addresses: prosso@prhtl.upv.es (Paolo Rosso), bosco@di.unito.it (Cristina Bosco), cambria@ntu.edu.sg (Erik Cambria), rossana@di.unito.it (Rossana Damiano), patti@di.unito.it (Viviana Patti)

Preprint submitted to Journal of Information Processing \& Management November 12, 2015 
poses special challenges, since it involves a deep understanding of natural language texts by the computational systems, from which we are still far (Cambria et al. 2013). Approaches enabling context- and concept-based analysis can lead to a better understanding of opinions expressed in textual data, so as to reduce the gap between unstructured information and structured machine-processable data. Moreover, the focus on multimedia contents calls for the development of new techniques for automatic extraction and classification of affective information, where both textual and extra-linguistic features can be exploited and combined via multimodal approaches (Mihalcea \& Strapparava, 2012, Sartori et al. 2015).

Another key aspect to consider in order to bring advancements in this field is related to the role of cognitive models of emotions. Affective models that encompass the expressive aims of social and expressive media, need to be defined and integrated into traditional techniques of analysis and information processing. In fact, generation and manipulation of produced contents, for distribution, categorization, browsing or visualization must be equipped with a proper model of their affective qualities - and of their reception by the users - especially when the practical goal of assembling and delivering to the users contents in a personalized way is pursued. In this context, studying emotion representations that enable semantic metadata processing is also an important issue to face in order to foster the interoperability and integration of affect processing tools.

In light of these considerations, this special issue focuses on the presentation and discussion of a set of novel computational approaches to the analysis of emotion and sentiment in social and expressive media.

\section{The ESSEM special issue}

For this special issue, we received twenty one articles. As a result of the review process, twelve papers were selected for inclusion in the special issue. The list of papers mirrors the positive acceptance of the focus on social and expressive media by the research community interested in emotion and sentiment analysis. Social and expressive media are evenly represented in the selected articles, with a number of papers that apply sentiment analysis techniques to different types of social media contents, and papers that specifically address expressive media, where emotion models and sentiment analysis techniques are applied to audiovisual media and digital artworks.

A first group of papers focussing on new approaches to sentiment analysis and opinion mining on social media texts - ranging from Twitter to Amazon reviews and YouTube comments - encompasses the works by Saif et al. (2015), Fersini et al. (2015), Xia et al. (2015), Severyn et al. (2015).

In "Contextual Semantics for Sentiment Analysis of Twitter" by Hassan Saif, Yulan He, Miriam Fernandez and Harith Alani a lexicon-based approach for sentiment analysis on Twitter is described (Saif et al., 2015). A model for describing the context of tweets is proposed. The model takes into account several aspects and their interrelations, and allows for the detection of sentiment at both entity-level and tweet-level. 
Elisabetta Fersini, Enza Messina and Federico Pozzi in their paper "Expressive signals in social media languages to improve polarity detection" (Fersini et al., 2015) investigate the impact of expressive signals, such as adjectives, pragmatic particles and expressive lengthening, on sentiment polarity classification on Twitter. Expressive signals have been used to enrich the feature space of baseline and ensemble classifiers. Experimental results show that only adjectives play a fundamental role as expressive signal.

In "Polarity Shift Detection, Elimination and Ensemble: A Three-stage Model for Document-level Sentiment Analysis" Rui Xia, Feng Xu, Jianfei Yu, Yong Qi and Erik Cambria propose a three-stage cascade model in order to address the polarity shift problem in the context of document-level sentiment classification (Xia et al. 2015). The results of a range of experiments on a dataset of reviews from Amazon.com illustrate that the proposed approach outperforms several alternative methods for dealing with the problem at issue.

Aliaksei Severyn, Alessandro Moschitti, Olga Uryupina, Barbara Plank and Katja Filippova propose a model for carrying out opinion mining on YouTube comments. They evaluate the effectiveness of their proposal on two languages: English and Italian (Severyn et al., 2015). In particular, they propose kernel methods applied to a robust shallow syntactic structure and show that the approach outperforms other basic models on cross-domain settings. A further relevant contribution of this work is to make a YouTube corpus (Italian and English) available to the research community.

Guerini \& Strapparava (2015) and Celli et al. (2015) give a closer look at the role of affect in the diffusion of information, a line of research that is relevant to media platforms in general. In particular, in "Why Do Urban Legends Go Viral?" Marco Guerini and Carlo Strapparava focus on urban legends, a kind of viral deceptive texts, in between credible and incredible (Guerini \& Strapparava, 2015). They explore the effectiveness of features related with the intuition that urban legends should mimic the details of news to be credible, while they should be emotional like a fairy tale to go viral. They present machine learning experiments showing that it is possible to recognize an urban legend using these features.

Then, in the paper "In the Mood for Sharing Contents: Emotions, Personality and Interaction Styles in the Diffusion of News" Fabio Celli, Arindam Ghosh, Firoj Alam, and Giuseppe Riccardi investigate how personality types and communication styles of Twitter users are related to the selection of contents to share in Twitter, affecting the diffusion of a positive or negative mood among users (Celli et al. 2015). They developed an aligned corpus of Tweets and news articles from Corriere.it - an Italian daily newspaper- with gold standard mood, and propose a classification model to automatically predict Twitter users that share positive and negative moods.

Another group of papers includes the works by Aljanaki et al. (2015), Scharl et al. (2015) and Bertola \& Patti (2015), which mainly address the role of emotions in expressive media, dealing with the dissemination and reception of audiovisual media and digital artworks. Notice that the social media and expressive media categories often overlap, thus confirming the need for an integrated 
approach to address affect related issues in the age of social networks. The work by Arno Scharl, Alexander Hubmann-Haidvogel, Alistair Jones, Daniel Fischl, Ruslan Kamolov, Albert Weichselbraun and Walter Rafelsberger, for instance, fits into both groups by joining video games as topic and social media as the context: in "Analyzing the Public Discourse on Works of Fiction Detection and Visualization of Emotion in Online Coverage about HBO's Game of Thrones" (Scharl et al. 2015) they propose an online platform, where affective and factual knowledge extracted from news and social media coverage on the tv series "Game of Thrones" is exploited in order to populate an interactive dashboard with trend charts and synchronized visual analytics. Positive and negative sentiments are computed automatically, shedding light on the perception of actors and new plot elements.

In "Studying emotion induced by music through a crowdsourcing game" Anna Aljanaki, Frans Wiering and Remco C. Veltkamp present the development of a new publicly available dataset of 400 musical excerpts from four genres annotated with induced emotion, with the twofold aim to reflect on models of musical emotions and to create a ground-truth to be used in training automatic music emotion recognition systems (Aljanaki et al. 2015). Data were collected by using an online "game with a purpose". Emotional annotation was based on the domain-specific Geneva Emotional Music Scale model (Zentner et al., 2008).

In "Ontology-based Affective Models to Organize Artworks in the Social Semantic Web" Federico Bertola and Viviana Patti focus on detecting emotions elicited by artworks via analysis of social tags (Bertola \& Patti, 2015). Elicited affective information refers to an ontology of emotions based on the Plutchik's model. Ontologies, linked data and affective lexicons are combined in a novel framework where emotion-driven organization and access to online art collections is enabled. The framework is evaluated against a dataset of tagged multimedia artworks from the ArsMeteo Italian art portal (http: //www.arsmeteo.org) via a user study.

Last, a group of papers focus, on the one hand, on the impact of emotions and sentiment information on different tasks, such as personalized tag-based search (Xie et al., 2015) and author profiling (Rangel \& Rosso, 2015), and on the other hand on representing emotions in ontologies, coping with the need for established, shared solutions for the affective analysis of social and expressive media (Sanchez-Rada \& Iglesias, 2015).

In "Incorporating Sentiment into Tag-based User Profiles and Resource Profiles for Personalized Search in Folksonomy" Haoran Xie, Xiaodong Li, Tao Wang, Raymond Y.K. Lau, Tak-Lam Wong, Li Chen, Fu Lee Wang and Qing $\mathrm{Li}$ investigate the integration of sentiment information to address the problem of the personalized tag-based search in collaborative tagging systems (Xie et al., 2015). Experiments prove that sentiment-based information can significantly improve the performance of personalized search in folksonomy. In addition, a comparison of the influence of different sentiment corpora is developed which indicates SenticNet (http://sentic.net) as the most prominent knowledge base to boost the performance.

The article "On the Impact of Emotions on Author Profiling" by Francisco 
Rangel and Paolo Rosso investigates the impact of emotions on author profiling (Rangel \& Rosso, 2015). The authors integrate the six basic emotions of Ekman (Ekman, 1972) within a novel graph-based approach and propose a model for identifying gender and age in the Spanish partition of the PAN-AP-13 corpus, by achieving comparable results to the best performing systems of the PAN Lab of CLEF 2013 (http://pan.webis.de/).

Finally, the work "Onyx: A Linked Data Approach to Emotion Representation" by J. Fernando Sánchez-Rada and Carlos A. Iglesias presents a new semantic vocabulary, called Onyx, to describe in a unified framework emotion analysis processes and results, by using semantic web and linked data technologies in order to connect results from different providers and applications (Sanchez-Rada \& Iglesias, 2015). The issue of integrating semantic web representations of lexical resources for sentiment analysis is also addressed.

\section{Concluding remarks}

Selected articles are a valuable and varied representation of approaches facing the challenges of processing the affective information in social and expressive media. The proposals tackle with automatic extraction and classification of the affective information not only in textual, but also in multimedia contents, with a special attention on the exploitation of psychologically grounded models of affect. They cope with a range of interesting tasks, in different domains - also related to art - where the impact of emotions and sentiment analysis has proven important in order to improve the understanding of the social and expressive media data.

A variety of social media textual datasets, mostly in English, have been processed - but, interestingly, some approaches considered texts in Italian and Spanish. Furthermore, in many cases, authors contributed with the development of new corpora annotated with affective labels related to sentiment, fine-grained emotions or moods, depending on the affective model of interest. This creates a ground-truth ready to be used in training new automatic systems: a valuable source of material to cross-validate approaches and to foster future research in the field of emotion and sentiment in social and expressive media.

\section{Aknowledgments}

We would like to thank the Editor in Chief Fabio Crestani and all authors for their valuable contributions. In addition, we are deeply grateful to the reviewers that helped with the decision process, and contributed with excellent reviews to make this issue possible: Valerio Basile, Enrico Blanzieri, Paula Carvalho, Alberto Díaz, Andrea Esuli, Davide Eynard, María Teresa Martín Valdivia, Rada Mihalcea, Manuel Montes-y-Gomez, Preslav Nakov, Malvina Nissim, David Eduardo Pinto-Avendaño, Daniele Radicioni, Francisco Rangel, Antonio Reyes, Sara Rosenthal, Irene Russo, Björn Wolfgang Schuller, Vivek Kumar Singh, Mike Thelwall, José Antonio Troyano, Alessandro Valitutti, Tony Veale, Gualtiero Volpe, Wei Wei and Bei Yu. 
Paolo Rosso has been partially funded by the WIQ-EI IRSES project (Grant No. 269180) within the EC FP7 Marie Curie People Framework and by the DIANA-APPLICATIONS - Finding Hidden Knowledge in Texts: Applications project (TIN2012-38603-C02-01). The last phase of the work of Viviana Patti was carried out at the Universitat Politècnica de València in the framework of a three-month fellowship of the University of Turin within the World Wide Style (WWS) Program, Second Edition, co-funded by Fondazione CRT.

\section{References}

Aljanaki, A., Wiering, F., \& Veltkamp, R. C. (2015). Studying emotion induced by music through a crowdsourcing game. Information Processing 85 Management, (pp. -). URL: http://www.sciencedirect.com/science/article/ pii/S0306457315000424

Bertola, F., \& Patti, V. (2015). Ontology-based affective models to organize artworks in the social semantic web. Information Processing $\& 3$ Management, (pp. -).

Cambria, E., Schuller, B., Xia, Y., \& Havasi, C. (2013). New avenues in opinion mining and sentiment analysis. IEEE Intelligent Systems, 28, 15-21.

Celli, F., Ghosh, A., Alam, F., \& Riccardi, G. (2015). In the mood for sharing contents: Emotions, personality and interaction styles in the diffusion of news. Information Processing 83 Management, (pp. -). URL: http: //www.sciencedirect.com/science/article/pii/S030645731500103X.

Ekman, P. (1972). Universals and Cultural Differences in Facial Expressions of Emotion. University of Nebraska Press.

Fersini, E., Messina, E., \& Pozzi, F. (2015). Expressive signals in social media languages to improve polarity detection. Information Processing 83 Management, (pp. -). URL: http://www.sciencedirect.com/science/article/ pii/S0306457315000497

Guerini, M., \& Strapparava, C. (2015). Why do urban legends go viral? Information Processing 85 Management, (pp. -). URL: http://www. sciencedirect.com/science/article/pii/S0306457315000540

Mihalcea, R., \& Strapparava, C. (2012). Lyrics, music, and emotions. In Proceedings of the 2012 Joint Conference on Empirical Methods in Natural Language Processing and Computational Natural Language Learning EMNLPCoNLL '12 (pp. 590-599). Stroudsburg, PA, USA: Association for Computational Linguistics. URL: http://dl.acm.org/citation. cfm?id=2390948. 2391015

Rangel, F., \& Rosso, P. (2015). On the impact of emotions on author profiling. Information Processing $\&$ Management, (pp. -). URL: http://www. sciencedirect.com/science/article/pii/S0306457315000783 
Saif, H., He, Y., Fernandez, M., \& Alani, H. (2015). Contextual semantics for sentiment analysis of twitter. Information Processing 83 Management, (pp. -). URL: http://www.sciencedirect.com/science/article/ pii/S0306457315000242.

Sanchez-Rada, J. F., \& Iglesias, C. A. (2015). Onyx: A linked data approach to emotion representation. Information Processing \&3 Management, (pp. -). URL: http://www.sciencedirect.com/science/article/ pii/S030645731500045X

Sartori, A., Yan, Y., Özbal, G., Akdag Salah, A. A., Salah, A. A., \& Sebe, N. (2015). Looking at mondrian's victory boogie-woogie: What do I feel? In Proceedings of the Twenty-Fourth International Joint Conference on Artificial Intelligence, IJCAI 2015, Buenos Aires, Argentina, July 25-31, 2015 (pp. 2503-2509).

Scharl, A., Hubmann-Haidvogel, A., Jones, A., Fischl, D., Kamolov, R., Weichselbraun, A., \& Rafelsberger, W. (2015). Analyzing the public discourse on works of fiction - detection and visualization of emotion in online coverage about hbo's game of thrones. Information Processing 83 Management, (pp. -). URL: http://www.sciencedirect.com/science/article/ pii/S0306457315000278

Severyn, A., Moschitti, A., Uryupina, O., Plank, B., \& Filippova, K. (2015). Multi-lingual opinion mining on youtube. Information Processing 83 Management, (pp. -). URL: http://www.sciencedirect.com/science/article/ pii/S0306457315000400

Silvia, P. J. (2005). Emotional Responses to Art: From Collation and Arousal to Cognition and Emotion. Review of General Psychology, 9, 342-357.

Xia, R., Wang, T., Yu, J.-F., F. Xu, Y. Q., \& Cambria, E. (2015). Polarity shift detection, elimination and ensemble: A three-stage model for document-level sentiment analysis. Information Processing $\mathscr{E}$ Management, (pp. -).

Xie, H., Li, X., Wang, T., Lau, R. Y., Wong, T.-L., Chen, L., Wang, F. L., \& Li, Q. (2015). Incorporating sentiment into tag-based user profiles and resource profiles for personalized search in folksonomy. Information Processing ES Management, (pp. -). URL: http://www.sciencedirect.com/science/ article/pii/S0306457315000394.

Zentner, M., Grandjean, D., \& Scherer, K. (2008). Emotions evoked by the sound of music: Characterization, classification, and measurement. Emotion, $8,494-521$. 